\title{
Elfe XX-XXI
}

ELFe XX-XXI

Études de la littérature française des XXe et XXIe siècles

$9 \mid 2020$

Dire et lire les vulnérabilités contemporaines

\section{Entretien avec Sandra Laugier (Alexandre Gefen)}

\section{Sandra Laugier et Alexandre Gefen}

\section{OpenEdition}

Journals

Édition électronique

URL : http://journals.openedition.org/elfe/1748

DOI : $10.4000 /$ elfe. 1748

ISSN : 2262-3450

Éditeur

Société d'étude de la littérature de langue française du XXe et du XXle siècles

\section{Référence électronique}

Sandra Laugier et Alexandre Gefen, «Entretien avec Sandra Laugier (Alexandre Gefen) », Elfe XX-XXI

[En ligne], 9 | 2020, mis en ligne le 20 septembre 2020, consulté le 24 septembre 2020. URL : http:// journals.openedition.org/elfe/1748; DOI : https://doi.org/10.4000/elfe.1748

Ce document a été généré automatiquement le 24 septembre 2020.

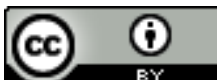

La revue Elfe XX-XXI est mise à disposition selon les termes de la Licence Creative Commons Attribution 4.0 International. 


\title{
Entretien avec Sandra Laugier (Alexandre Gefen)
}

\author{
Sandra Laugier et Alexandre Gefen
}

\section{Alexandre Gefen : D'où vient en philosophie le concept de vulnérabilité?}

Sandra Laugier: La vulnérabilité humaine n'est pas une idée nouvelle et elle a été discutée dans diverses pensées contemporaines, par exemple les théories de la reconnaissance. Le risque pour la vie (d'être blessé, de disparaître) est présent dans toutes les pensées politiques. Mais le thème central en est la mortalité. Or c'est une nouvelle anthropologie qui émerge avec la vulnérabilité, car elle concerne les vivants ; et pas seulement la possibilité de la blessure physique, mais morale.

La vulnérabilité a ainsi acquis une certaine fortune ces dernières décennies dans le champ de la réflexion d'abord morale, puis politique, souvent associée à d'autres notions comme celles de dépendance, de précarité ou de fragilité. Elle a émergé avec la prise de conscience $d u$ fait que la pensée politique dominante, libérale (globalement issue de la théorie de la justice de Rawls, avec des variantes importantes bien sûr) était en grande partie aveugle à un certain type d'injustice et de détresse : des injustices si radicales que les victimes en sont exclues de la communauté rationnelle d'élaboration des critères de la justice, des injustices dues à l'interdépendance des sujets politiques, qui fait que l'autonomie et la liberté des uns, si valorisées dans la pensée libérale, se construit aux dépens d'autres.

La vulnérabilité renvoie donc à une condition anthropologique : nous sommes « tous vulnérables ", et c'est cette dimension qui est la première réalité, en rupture avec les différents impératifs ou idéaux moraux qui constituent la morale classique : liberté, autonomie, équité...

La notion de vulnérabilité enfin a été promue et approfondie par le développement de l'éthique du care. Le care n'a pas découvert la vulnérabilité ou la fragilité, thèmes déjà développés dans bien des réflexions morales contemporaines (Judith Butler, Giorgio Agamben, Martha Nussbaum) ; mais il met la vulnérabilité au cœur de la 
morale, et en fait aussi une arme politique (voir le livre de Marie Garrau, Politiques de la vulnérabilité).

Alexandre Gefen : Dans quelles situations notre vulnérabilité s'expose-elle particulièrement $?$

Sandra Laugier : L'idée de vulnérabilité s'impose, à première vue lorsqu'on pense au petit enfant, au grand âge et à la maladie... Elle est liée à l'idée de protection et est évidente pour les personnes qui nécessitent pour être en vie des soins constants. On en a eu des exemples avec des malades en soins intensifs.

Le concept contemporain de vulnérabilité va au-delà de ces situations; il y a des situations de grande vulnérabilité, "radicale ", comme la pandémie ou la catastrophe, où des populations entières se trouvent " à la merci ", sans défense, souvent parce qu'elles cumulent des vulnérabilités, sociales (pauvreté), géographiques (zone dangereuse), politiques (guerres), sanitaires... Le concept d'inégalité environnementale traduit cela. Les désastres et catastrophes ébranlent les formes de vie entières et non seulement tuent, mais créent des conditions de vie d'un dénuement extrême, où les idées de bien-être ou d'accomplissement sont totalement exclues et où la vie n'est pas digne d'être vécue. C'est ce qu'entend Amartya Sen avec son concept de « capabilité ».

Alexandre Gefen: Sommes-nous vraiment, pour reprendre le titre de l'un de vos livres, " tous vulnérables »? Certains ne le sont-ils pas plus que d'autres?

Sandra Laugier: Mais oui, la vulnérabilité est partagée par tous: "Les gens vulnérables n'ont rien d'exceptionnel ", pour reprendre le joli titre de Patricia Paperman (2005), qui reprenait un titre de film, Les gens normaux n'ont rien d'exceptionnel (Laurence Ferreira Barbosa, 1993).

Bien sûr, il y a des degrés dans la vulnérabilité et des personnes peuvent sembler moins vulnérables que d'autres, parce que protégées ; notamment si l'on envisage les situations extrêmes où la survie est en cause. Mais justement l'éthique du care vise à montrer la vulnérabilité de chacun, à en finir avec l'abstraction éthico-politique de l'individu indépendant et autonome, qui ne serait vulnérable qu'au grand âge et dans la petite enfance, ou en cas d'accident de parcours. Comme on l'a vu lors du confinement, la plupart des personnes privilégiées sont loin d'être autonomes et vivent en s'appuyant sur toutes sortes de ressources : les services de santé, mais aussi les services de ceux qui font le ménage, fournissent la nourriture, livrent des objets et ont continué d'ailleurs durant la crise. Chacun a pu se retrouver dépourvu à divers degrés. Plus les personnes se sentent autonomes, plus elles s'appuient sur un système qui est mis à leur " service ". Le care nous rappelle que nous avons besoin d'autres pour satisfaire nos besoins, et c'est le cas de tout le monde, sauf justement des plus vulnérables qui ne peuvent compter que sur eux-mêmes. D'où la prudence nécessaire quand il s'agit de vulnérabilité, car il ne faudrait pas, comme cela s'est vu avec d'autres valorisations ethnocentrées, "distinguer " des vulnérables qui sont toujours les autres - les femmes, les groupes racisés ou socialement dévalorisés.

Alexandre Gefen: Vous êtes spécialiste de Stanley Cavell, qui a réfléchi sur notre vulnérabilité dans le langage...

Sandra Laugier: L'humain est vulnérable et cette vulnérabilité passe aussi par l'expression. La capacité de langage est intégrée dans la vulnérabilité, et au-delà même de l'humain l'expression de la douleur est la marque du vulnérable. Au centre 
de l'éthique du care, il y a notre capacité (notre disposition) à l'expression morale laquelle, comme l'ont montré de diverses manières Stanley Cavell et Charles Taylor, s'enracine dans une forme de vie, au sens d'un agrégat à la fois naturel et social de formes d'expression et de liens à autrui. L'anthropologue Veena Das connecte ainsi l'idée de forme de vie à cette vulnérabilité de l'expressivité humaine, qui expose le sujet. La conversation, l'échange humain est aussi un lieu de haute vulnérabilité, même si c'est aussi un cadre de consolidation de la société : on risque toujours de s'exprimer de travers, de créer des ruptures. La philosophie du langage ordinaire de J. L. Austin et de Wittgenstein a mis en évidence cette vulnérabilité de l'expression humaine. Une telle vulnérabilité est au cœur de la théorie des actes de langage (2004). Un énoncé performatif, s'il manque son but (pour diverses raisons: émission, contexte, réception) est malheureux, manqué. La pragmatique austinienne déplace le faux vers le raté. Cette possibilité ressortit à la dimension d'acte de toute parole : c'est parce que le langage est acte qu'il échoue. Le malheur de l'acte de langage est le malheur possible de toute action humaine. C'est cette vulnérabilité propre de l'action qui crée les échecs, et l'activité qui leur est symétriquement associée : celle des excuses, qui est un sujet infini de réflexions pour Austin et Cavell. Le domaine de l'excuse délimite, et différencie finement entre elles, les erreurs humaines, c'est-àdire les mises en œuvre de la vulnérabilité propre de l'humain en tant qu'il risque d'échouer dès qu'il agit. La vulnérabilité du langage est dans la vulnérabilité de l'agentivité humaine.

Alexandre Gefen: La vulnérabilité est-elle uniquement individuelle ? À I'heure des pandémies et des régimes illibéraux, le concept peut-il être utilisé pour parler des sociétés et de la démocratie?

Sandra Laugier : La vulnérabilité est collective avant d'être individuelle, car il s'agit souvent de groupes, de catégories sociales que l'on rend vulnérables, la vulnérabilité n'est pas un donné puisque la question est de savoir comment on la pallie. Par exemple dans la pandémie du COVID, il y a des différences dans les pays pour différentes raisons, mais on a pu constater une surmortalité de certaines catégories dans des pays comme les États-Unis, où le taux de mortalité des Noirs atteints est 2,5 fois supérieur à celui des Blancs, à cause d'une mauvaise santé initiale elle-même liée à des conditions de vie déplorables (pauvreté, cadre malsain, négligence des autorités etc.). On a là un bon exemple de vulnérabilité collective. On peut penser aussi en France aux femmes qui assuraient des fonctions de care, que ce soit dans les services de santé ou dans les commerces, et qui ont été rendues vulnérables par l'absence de protection dans les transports et au travail, et en général par des conditions de vie épuisantes et dégradantes.

Plus généralement, la vulnérabilité est un concept politique, car une société morale se soucie du bien-être des citoyens et donc doit aussi prendre en compte l'ensemble des personnes qui s'occupent d'eux. C'est en reconnaissant la société dans son ensemble comme vulnérable qu'on peut commencer à considérer à sa juste valeur la contribution de celles et ceux qui la font vivre.

Pour les régimes autoritaires que vous mentionnez, ce qui les caractérise de façon assez flagrante, c'est qu'ils sont opposés à toute forme de care et n'admettent pas la vulnérabilité, surtout la leur. Ils récusent la nécessité pour un gouvernement de prendre soin des plus vulnérables, et en font même un principe politique. Enfin on peut parler de vulnérabilité collective des pays du Sud, notamment par la « fuite du 
care » qui conduit de très nombreuses femmes et hommes à quitter leur pays pour venir dans les pays riches s'occuper de nos vulnérabilités, ce qui fragilise les structures de care dans leur pays, où ils doivent laisser leur famille.

Alexandre Gefen : Qui prend en charge nos vulnérabilités ? Comment intervient la notion de care ?

Sandra Laugier: Le care c'est précisément le travail de prise en charge des vulnérabilités, car c'est la réponse aux besoins, qui peuvent être variables. Il renvoie à une réalité bien ordinaire : le fait que des gens s'occupent d'autres, s'en soucient et ainsi veillent au fonctionnement du monde. Le care est aussi un outil d'analyse des conditions historiques qui ont favorisé une division du travail moral en vertu de laquelle les activités de soins et de soutien ont été socialement et moralement dévalorisées. Il porte une revendication fondamentale concernant l'importance de ces fonctions pour la vie humaine et de la position sociale et morale des caregivers. L'éthique du care s'est ainsi révélée plus largement un projet de société visant à mettre la réflexion sur les vulnérabilités et les liens au centre non seulement d'un travail sur la vie morale, mais également d'un travail sur la définition du politique. Le concept de care a ainsi joué un rôle de révélateur social et politique de la vulnérabilité et a aussi révélé depuis longtemps une réalité qui se fait jour maintenant: que les personnes vulnérables ne sont pas seulement les malades et ceux qu'on soigne, mais aussi les personnes qui s'occupent d'eux et sont particulièrement vulnérables (dans leurs conditions de travail, leur niveau social, et du fait du système mondial de l'immigration des travailleurs de care des pays du Sud vers ceux du Nord.) Le fait qu'on parle maintenant de prendre soin des soignants est une bonne chose, même si c'est tard.

Alexandre Gefen : Quelle place accordez-vous aux arts, en particulier à la littérature, pour dire la vulnérabilité ?

Sandra Laugier: La littérature a été de longue date le lieu d'expression de la vulnérabilité individuelle et c'est ce qui en fait l'attractivité et la richesse. C'est aussi elle qui a donné vie et place à toutes sortes de catégories sociales négligées par l'histoire et la politique, toutes les petites gens ou vies minuscules. C'est la lecture aussi qui prend soin de nous et de nos fragilités. Mais pour moi le cinéma a joué un rôle aussi essentiel dans l'expression de la vulnérabilité, par la présentation des corps et expressions à l'écran, des personnes en situation de dénuement, et ce dès ses origines (je pense à Chaplin) et dans ses développements les plus intéressants (les frères Dardenne, par exemple).

\section{RÉSUMÉS}

Sandra Laugier revient dans cet entretien sur les fondements anglo-saxons de la théorie de la vulnérabilité et notamment sur l'importance de Stanley Cavell, sa dimension anthropologique comme sociale. La vulnérabilité de l'expression humaine, celle de 
l'être en tant qu'il est exposé aux ratages de la communication résonne dans la responsabilité de la parole littéraire.

Sandra Laugier is Professor of Philosophy at the University of Paris 1 Panthéon-Sorbonne, she has contributed to the popularization in France of the ordinary language philosophy, gender studies and the ethics of care. In this interview, she discusses the foundations of the theory of vulnerability and in particular the importance of Stanley Cavell, its anthropological as well as social dimension.

\section{INDEX}

Mots-clés : vulnérabilité

Keywords : vulnerability

\section{AUTEURS}

\section{SANDRA LAUGIER}

Sandra Laugier est professeure de philosophie à l'Université Paris 1 Panthéon-Sorbonne. Elle a contribué à populariser en France la philosophie du langage ordinaire, les études de genre et la philosophie du care. On lui doit notamment en 2012 Tous vulnérables ? Le care, les animaux, l'environnement (Payot) et en 2013 Face au désastre. Le care, la folie et les grandes détresses collectives (S. Laugier, V. Das, S. Pandolfo, A. Lovell [éd.] Ithaque).

\section{ALEXANDRE GEFEN}

Alexandre Gefen est Directeur de Recherche au CNRS (UMR Thalim/Université Paris 3 - Sorbonne nouvelle). Directeur Adjoint Scientifique de l'Institut des Sciences Humaines et Sociales du CNRS, fondateur de Fabula.org, il travaille sur la théorie littéraire, les littératures contemporaines, les écritures et les humanités numériques. Il est par ailleurs critique littéraire. Dernières parutions : Vies imaginaires de la littérature française, Paris, Gallimard, 2014 ; Art et émotions, Armand Colin, 2015 ; Inventer une vie. La fabrique littéraire de l'individu, Les Impressions Nouvelles, 2015. Réparer le monde. La littérature française face au XXI' siècle, Corti, 2017. A paraître : L'idée de littérature. De l'art pour l'art aux écritures d'intervention, Corti, 2021. 